\title{
Efficacy and Safety of Glandular Flap Techniques in Surgical Treatment of Large Ductal Carcinoma in situ
}

\author{
Lutfi Dogan ${ }^{\mathrm{a}} \quad$ Mehmet Ali Gulcelik ${ }^{\mathrm{b}}$ \\ a Department of General Surgery, University of Health Sciences, Ankara Oncology Training and Research Hospital, \\ Ankara, Turkey; ${ }^{b}$ Department of General Surgery, University of Health Sciences, Gülhane Training and Research \\ Hospital, Ankara, Turkey
}

\section{Keywords}

Breast-conserving surgery · Ductal carcinoma in situ • Oncoplastic surgery $\cdot$ Re-excision

\begin{abstract}
Background: It is well known that full segmentary resection can be performed using oncoplastic surgery (OPS) techniques, and the anatomic resection of the ductal system is possible. Therefore, the efficacy and safety of OPS should be investigated in the treatment of ductal carcinoma in situ (DCIS). Patients and Methods: Patients who were diagnosed as pure DCIS and underwent surgical treatment and followup were retrospectively evaluated. Patients who underwent OPS and conventional breast-conserving surgery (BCS) were included in the study. The number of patients who required an intervention after the surgery and had a relapse during the follow-up period was determined in both groups. $R \boldsymbol{e}$ sults: There were 45 patients in the OPS group and 138 patients in the BCS group. The mean tumor size was larger in patients in the OPS group ( $36 \pm 12 \mathrm{~mm}$ vs. $24 \pm 8 \mathrm{~mm}, p=$ 0.02). Six (12.7\%) patients were reoperated in the OPS group. Of these, 4 were re-excisions and 2 were mastectomies. In this group, breast conservation was possible in 45 (95.7\%) patients. Thirty-nine (27\%) patients were reoperated in the $\mathrm{BCS}$ group. Of these, 23 were re-excisions, and 16 were mastectomies. In this group, breast conservation was possible in $126(88.7 \%)$ patients $(p=0.02)$. There was no significant dif-
\end{abstract}

ference between the groups in terms of 5-year cumulative local recurrence rates. While the 5-year local recurrence-free survival rate was $93.3 \%$ in the OPS group, it was $90.8 \%$ in the BCS group. Conclusion: This study provided evidence that OPS can be used safely in the surgical treatment of DCIS by reducing re-excision and completion mastectomy rates compared to BCS.

(c) 2020 S. Karger AG, Basel

\section{Introduction}

The ducts in the terminal ductal-lobular units of the breast are covered with a single layer of epithelium. Ductal carcinoma in situ (DCIS) of the breast represents neoplastic cells which do not exceed the basement membrane of the terminal ductal lobular units. DCIS is a precursor lesion with the potential to progress to invasive disease. As the number of screening programs increases and imaging methods develop in parallel with technology, the proportion of patients diagnosed with DCIS among all breast cancer cases has increased all over the world. The main purpose of screening programs is to detect the tumor either in situ or smaller than $1 \mathrm{~cm}$. Even though DCIS rates among newly diagnosed breast cancers are lower than $5 \%$ in underdeveloped countries, this rate may reach $20-25 \%$ in developed countries [1]. 
DCIS represents a heterogeneous group of diseases. Structural changes, nuclear grade, and presence of necrosis can reveal the changes in DCIS [2]. DCIS involves the breast ductal system, and it tends to spread radially throughout the segment, so providing a more accurate surgical margin is more problematic than invasive tumors [3]. A robust surgical margin is the most important factor affecting survival [4]. Theoretically, the main purpose in the treatment of an entity which does not have the chance of regional and distant metastasis is to provide a full cure and then to conserve the patient's breast with acceptable cosmetic results. Approximately $20-30 \%$ of DCIS have extensive microcalcifications (MC) in mammography and these patients are treated with mastectomy and concomitant reconstruction [5]. Cosmetic results are often unsatisfactory when conventional breast-conserving surgery (BCS) techniques are used in patients who require extensive resections. Another issue when BCS is applied is inadequate surgical margins and need for reoperations. It is well known that oncoplastic surgery (OPS) allows wider resections with better cosmetic results and reduces reoperation rates in surgical treatment of invasive breast cancers [6].

OPS techniques in the treatment of patients with DCIS who have undergone or who are technically eligible for BCS are not as widely used as in invasive tumors. Utilizing OPS for bigger tumors and hard to reach locations increases simultaneously with extensive experience [7].

The aim of the study is to compare the oncoplastic glandular flap (volume displacement) techniques with respect to surgical margin safety, reoperation requirement, breast protection rates, complications, and long-term local control rates with conventional BCS.

\section{Materials and Methods}

Patients who were diagnosed as pure DCIS between 2012 and 2017 and underwent surgical treatment and follow-up in our clinic were retrospectively evaluated. Patients who underwent OPS and conventional BCS were included in the study. Patients who underwent mastectomy as the first treatment, for various reasons, especially for diffuse MC, were excluded from the study. In addition, patients treated for a component of an invasive tumor were also excluded from the study. Age, body mass index (BMI), menopausal status, tumor size, nuclear grade, presence of comedonecrosis, receptor status, diagnostic methods, extracted specimen volumes, distance to surgical margins, surgical techniques, wound complications that occurred in early term, duration of stay at the hospital, and times for starting the adjuvant therapy were recorded. The number of patients who required an intervention after the surgery and had a relapse during the follow-up period was determined in both groups. Local control and survival rates were determined.

Regardless of age, all patients had undergone mammography. Magnetic resonance imaging (MRI) was performed for preoperative localization when required. MRI was performed in patients with suspicious linear MC on mammographic examina- tion but whose breast density was heterogeneous (BIRADS 3) or severely dense (BIRADS4) and insufficient information was obtained on mammography. MRI-guided wire localization biopsy was performed in patients whose lesions were visualized only on MRI. Patients who presented with MC were diagnosed with wire-guided biopsy and those with masses were diagnosed with percutaneous trucut biopsy. Patients presenting with MC were either marked with wire for preoperative MC margins, or the skin projections of the margins were marked. Specimen radiographs were taken to investigate MC in these patients. Perioperative re-excisions were performed in patients with $\mathrm{MC}$ near the margins. Cavity shaving was not performed in any patients, but additional surgical margin resections were performed in cases where the surgical margins were clinically suspected. Tumor beds were marked with metallic clips. The surgical margin was accepted as positive if there was ink on the tumor on surgical margin as agreed at the 14 th St. Gallen International Breast Cancer Conference. In addition, surgical margins were grouped as 0-1 mm, 1-2 mm, and $>2 \mathrm{~mm}$. In patients diagnosed with percutaneous biopsy, the choice of sentinel lymph node biopsy was performed with the clinician's preference, with the suspicion of microinvasion in the final specimen. All patients were treated with conventional adjuvant radiotherapy ( 2 Gy/day) with a total of $50 \mathrm{~Gy}$, and boost dose radiotherapy was delivered to the tumor bed. Hormone therapy was delivered in patients with positive hormone receptors.

Specimen volumes were determined by multiplying the three dimensions of the specimen indicated in the pathology report. In the conventional BCS group, there were patients who had undergone lumpectomy. Level II volume displacement techniques applied in the OPS group were as follows: Racket technique, Roundblock, Batwing, inferior and superior pedicular reduction, radial and fusiform mammoplasty, and vertical mammoplasty. Wound complications were evaluated in two groups, as having minor or major complications. Seroma, hematoma, wound infection, delayed wound healing, and incisional wound dehiscence were evaluated in the minor group. Major serious complications such as incisional wound dehiscence and nipple necrosis were evaluated in the major group. Serous fluid collection, creating patient discomfort and tension, led to both incision and drainage; they were considered to be a seroma. Conversely, collections which caused hemorrhagic bruises on the skin were considered to be hematomas. Erythema, purulent discharge, localized temperature increases, cellulitis, pain, redness, tenderness (whether confirmed with wound culture or not) were considered to be wound infection. Simple incision dehiscence repaired by simple suturing was classified as minor, while repair of the whole incision in an operating theater was classified as major wound dehiscence. Wound dehiscence that heals without intervention was regarded as late wound healing.

Patients were invited for follow-up at 3-month intervals for the first 2 years and at 6-month intervals for the next 3 years. After 5 years, the patients were called at 12-month intervals. In addition to physical examinations, annual mammography and breast ultrasonography were performed as standard. Breast MRI was performed if necessary.

SPSS 25 statistical program was used for statistical analyses (IBM Corp., released 2017; IBM SPSS Statistics for Windows, version 25.0, Armonk, NY, USA). Mann-Whitney U test was used for comparisons between the groups. Quantitative data (patient and tumor characteristics, surgical margins proximities, reoperation rates, and results of surgical techniques) were evaluated by $\chi^{2}$ test. The Kaplan-Meier test was used to determine breast cancer recurrence rates and local and total survival time. 
Table 1. General characteristics of the patients

\begin{tabular}{lccc}
\hline & $\begin{array}{l}\text { OPS } \\
(n=47)\end{array}$ & $\begin{array}{l}\text { BCS } \\
(n=142)\end{array}$ & $\begin{array}{l}p \\
\text { value }\end{array}$ \\
\hline Age & $48 \pm 8$ & $49 \pm 7$ & \\
Tumor size, mm & $36 \pm 12$ & $24 \pm 8$ & 0.02 \\
BMI & $31 \pm 4$ & $27 \pm 3$ & 0.04 \\
Specimen volume, $\mathrm{cm}^{3}$ & $416 \pm 85$ & $175 \pm 41$ & 0.01 \\
Grade 3 & $64 \%$ & $60 \%$ & 0.2 \\
Comedonecrosis & $44 \%$ & $49 \%$ & 0.3 \\
ER (+) & $75 \%$ & $79 \%$ & 0.5 \\
PR (+) & $66 \%$ & $62 \%$ & 0.5 \\
Premenopausal & $44 \%$ & $42 \%$ & 0.3 \\
\hline
\end{tabular}

ER, Estrogen receptor; PR, progesterone receptor; BMI, body mass index.

\section{Results}

In the OPS group, a total of 47 interventions in 45 patients (bilateral tumor in 2 patients), and in the BCS group, 142 interventions in 138 patients (bilateral tumor in 4 patients) were performed. During this period, 84 patients who were excluded from the study had undergone mastectomy for DCIS. Of the 84 patients who underwent mastectomy, 26 had extensive microclassifications on preoperative mammography (31\%). The mean tumor size was $38 \pm 14 \mathrm{~mm}$. The mean BMI of the patients was $32 \pm$ 4. The rate of patients with comedonecrosis was $45 \%$ and the rate of grade 3 patients was $61 \%$. There was no difference between the patients who underwent mastectomy and OPS in terms of tumor and patient characteristics. There was no local recurrence in the patients who underwent mastectomy within the follow-up period.

There was no difference between the all groups in terms of age, menopause, nuclear grade, presence of comedonecrosis, and receptor status. The mean tumor size was larger in patients in the OPS group $(36 \pm 12 \mathrm{~mm}$ vs. $24 \pm 8 \mathrm{~mm})$. Twenty-eight patients who presented with $\mathrm{MC}$ were diagnosed with wire-guided biopsy. Nineteen patients with masses were diagnosed with percutaneous trucut biopsy in the OPS group. While the mean specimen volume in the OPS group was $416 \pm 85 \mathrm{~cm}^{3}$, it was $175 \pm 41 \mathrm{~cm}^{3}$ in the BCS group. The specimen volume in the OPS group was significantly higher than the BCS group $(p<0.01)$. In addition, the BMI of the patients in the OPS group was significantly higher than the BMI of the patients in the BCS group. General characteristics of the patients are shown in Table 1.

The most common technique used in the OPS group was the Racket technique, with 18 patients (38\%). Reduction mammoplasty was performed with flap technique in 11 patients with lower pedicle and in 7 patients with upper pedicle. Vertical mammoplasty was performed in 4 pa- tients, and radial or fusiform mammoplasty was performed in 3 patients. Donut technique was performed in 2 of 4 patients with periareolar localization, and Batwing technique was performed in the remaining 2 patients. In the OPS group, nipple and areola were excised in 7 patients (15\%) who underwent reduction mammoplasty and vertical mammoplasty. Nipple and areola excision were performed in 27 (19\%) patients in the BCS group. There was no difference between the techniques in terms of surgical margin positivity, re-excision and mastectomy rates. Techniques used in the OPS group are shown in Table 2.

The surgical margins were positive in 4 patients $(8.5 \%)$ in the OPS group. While the number of patients whose surgical margins were closer than $1 \mathrm{~mm}$ was 5 (10.6\%), the number of patients with surgical margins between $1-2 \mathrm{~mm}$ was $7(14.8 \%)$. In 31 patients $(66 \%)$, the surgical margins were more than $2 \mathrm{~mm}$. The surgical margins were positive in 24 (16.9\%) patients in the BCS group. While the number of patients with surgical margins closer than $1 \mathrm{~mm}$ was $31(21.8 \%)$, the number of patients between 1-2 $\mathrm{mm}$ was 49 (34\%). Thirty-eight patients $(26.7 \%)$ had a margin of more than $2 \mathrm{~mm}$. While the mean distance of the tumor to the surgical margin was 26 $\pm 8 \mathrm{~mm}$ in the OPS group, it was $17 \pm 6 \mathrm{~mm}$ in the BCS group. In the OPS group, the surgical margins were significantly wider. Six (12.7\%) patients were reoperated in the OPS group. Of these, 4 were re-excisions and 2 were mastectomies. In this group, breast conservation was possible in $45(95.7 \%)$ patients. Thirty-nine (27\%) patients were reoperated in BCS group. Of these, 23 were re-excisions and 16 were mastectomies. In this group, breast conservation was possible in $126(88.7 \%)$ patients. Breast conservation rates were significantly higher in the OPS group. The surgical margin proximities and reoperation characteristics in both groups are given in Table 3.

Sentinel lymph node biopsy was performed in 14 $(29.7 \%)$ patients in the OPS group and in $36(25.3 \%)$ in the BCS group. All sentinel lymph node biopsies were negative. The first 30-day morbidities revealed seroma in 4 patients, wound dehiscence in 4 patients, wound infection in 2 patients, and hematoma in 1 patient in the OPS group. The numbers were $6,4,4$, and 3 in the BCS group, respectively. There was no nipple necrosis in both groups. While the total wound complication rate was $23 \%$ in the OPS group, it was $12 \%$ in the BCS group. All patients were discharged on the first postoperative day in the BCS group. The median duration of stay at the hospital was 1.5 days in the OPS group. While the adjuvant radiotherapy was started after 27 days in the OPS group, it was 24 days in the BCS group, and no significant difference was found between the groups $(p>0.05)$.

After a mean follow-up period of 41 months, ipsilateral breast tumor recurrence (IBTR) was detected in $3(6.3 \%)$ patients in the OPS group. Of these, 2 were in situ and 1 was 
Table 2. Techniques used in the OPS group

\begin{tabular}{lllll}
\hline Surgical techniques & $n(\%)$ & Surgical margin $(+)$ & Re-excision & Mastectomy \\
\hline Racket technique & $18(38 \%)$ & 1 & 1 & 1 \\
Reduction with lower pedicle & $11(23.5 \%)$ & 1 & 1 & 1 \\
Reduction with upper pedicle & $7(14.8 \%)$ & & & \\
Vertical mammoplasty & $4(8.5 \%)$ & & 1 & \\
Radial-fusiform mammoplasty & $3(6.3 \%)$ & 1 & 1 \\
Donut & $2(4.2 \%)$ & 1 & & \\
Batwing & $2(4.2 \%)$ & & & \\
\hline
\end{tabular}

Table 3. Surgical margin proximities and reoperation characteristics in both groups

\begin{tabular}{lcll}
\hline & OPS $(n=47)$ & BCS $(n=142)$ & $p$ value \\
\hline SM $(+)$ & $4(8.5 \%)$ & $24(16.9 \%)$ & 0.01 \\
SM $<1 \mathrm{~mm}$ & $5(10.6 \%)$ & $31(21.8 \%)$ & 0.02 \\
SM $1-2 \mathrm{~mm}$ & $7(14.8 \%)$ & $49(34 \%)$ & 0.01 \\
SM $>2 \mathrm{~mm}$ & $31(66 \%)$ & $38(26.7 \%)$ & 0.01 \\
Median SM, mm & $26 \pm 8$ & $17 \pm 6$ & 0.01 \\
Reoperation & & & \\
$\quad$ Re-excision & $4(8.5 \%)$ & $23(16.1 \%)$ & 0.02 \\
$\quad$ Mastectomy & $2(4.2 \%)$ & $16(11.2 \%)$ & \\
Breast conservation & $45(95.7 \%)$ & $126(88.7 \%)$ & 0.01 \\
\hline
\end{tabular}

SM, surgical margin.

invasive cancer. In the BCS group, IBTR was detected in 12 (8.4\%) patients after a mean 43-month follow-up, and 2 of these were invasive cancers. There was no significant difference between the groups in terms of 5-year cumulative local recurrence rates. While the 5 -year local recurrence-free survival was $93.3 \%$ in the OPS group, it was $90.8 \%$ in the BCS group (95\% CI 0.54-3.63; $p=0.71$ ) (Fig. 1).

\section{Discussion}

As the rate of DCIS increases among breast cancers, surgeons will have to treat more patients with DCIS. Mastectomy and simultaneous total reconstruction are still the most prevalent treatment options in patients with multicentric spread and diffuse MC. Mastectomy is performed in approximately $30-35 \%$ of the patients, but practically, not all DCIS patients who have undergone mastectomy have diffuse MCs or have multicentric disease [5]. DCIS involves a ductal system and spreads radially away from the nipple or towards the nipple [8]. Although it is expected to spread along an anatomical segment, it may have a hopping spread pattern due to the complex anatomical structure of the ductal system. In other words, DCIS can progress along the segments, leaving areas without involvement [9]. In asymptomatic

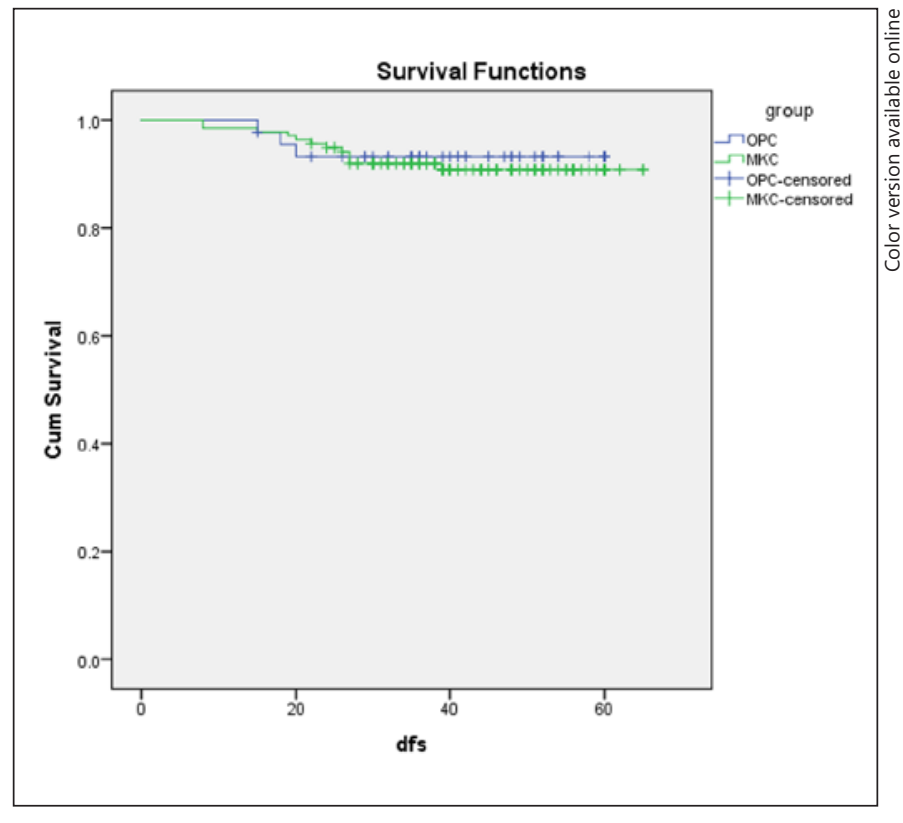

Fig. 1. Five-year local recurrence-free survival curves.

women joining screening programs, DCIS is usually detected as MC in mammographies. It is much harder to predict the spread pattern of DCIS which does not develop any masses, meaning that surgical safety margin is harder to provide in DCIS when compared to invasive tumors [10]. If there is tumor at the inked margin, the surgical margin is accepted as positive and in spite of the radiotherapy, recurrence is twice in these patients. Approximately half of these recurrences are invasive tumors [11]. This complicated situation involving the surgical margin leads surgeons to perform wider resections and mastectomy with conventional methods. In conventional BCS, resecting more than $10 \%$ of the mammary tissue may cause an unfavorable cosmetic outcome in approximately $50 \%$ of patients [12]. As glandular tissue replacement is performed in OPS and re-excision is difficult to perform when necessary, use of OPS in the surgical treatment of DCIS has not reached the desired levels.

A significant number of patients with positive surgical margins undergo mastectomy in the second session after 
conventional BCS. OPS is generally performed to correct the deformity in patients with DCIS who have undergone conventional BCS, even if there is adequate surgical margin [13]. However, it is well known that we can resect more tissues using the OPS techniques, and OPS can be performed on bigger tumors as the experience of the operator increases. In addition, full segmentary resection can be performed by OPS techniques, and anatomic resection of the ductal system towards the nipple is possible. Therefore, the efficacy and safety of OPS should be investigated.

In 2016, the American Society of Clinical Oncology (ASCO), Society of Surgical Oncology (SSO), and American Society for Radiation Oncology (ASTRO) published a joint declaration and recommended a 2-mm surgical margin for DCIS. However, this recommendation is not based on concrete evidence. In addition, re-excision is not recommended for patients whose surgical margins are less than $2 \mathrm{~mm}$. Other factors increasing the risk of recurrence, such as comedonecrosis, high grade, young age, and hormone receptor negativity, should be considered [14]. On the other hand, physicians have come to an agreement that the concept of "no ink on tumor," which was accepted at the 14th St. Gallen International Breast Cancer Conference for invasive tumors, is also valid for DCIS [15].

In our study, we showed that OPS is superior to BCS in terms of providing surgical margins distant to $2 \mathrm{~mm}$, even though OPS can be performed on bigger tumors, and larger tissues can be removed. As a result, compared to BCS, reoperation rates are lower and breast conservation rates are higher in OPS. In BCS, re-excision rates have been reported around 30\% [16]. In our BCS group, we have encountered similar rates. It should be kept in mind that while re-excisions increase patient anxiety and treatment costs, they also worsen the cosmetic results [17].

In a retrospective series of 39 patients investigating the efficacy of level II oncoplastic techniques in DCIS [18], which allowed resection of $20-50 \%$ of breast tissue, the mean surgical margin has been determined as $11 \mathrm{~mm}$. Negative surgical margins were achieved in all patients, provided that re-excision was performed in 3 patients. In this study, only 3 patients have presented with seroma. In a French study [10], including 68 patients with an average tumor size of $34 \mathrm{~mm}$, surgical margin positivity has been detected in 10 (14\%) patients and 5-year cumulative local recurrence rate is $5.5 \%$. In our study, even though the surgical margin positivity is lower, local control rates are similar.

The local recurrence rates which have been reported after BCS for DCIS are higher than 10\% [19]. In our study, there was no significant difference between the groups in terms of local recurrence. Increased efficacy of radiotherapy and hormone therapy may be the possible reasons for this, or as the follow-up time increases, there may be a difference between the groups.

Treatment of Large Ductal Carcinoma in situ
In the study by Song et al. [20], which recruited 28 patients, when positive surgical margin was accepted as 1 $\mathrm{mm}$, the necessity of re-excision has been reported as $36 \%$. In this series, where only one local relapse was detected, the minor wound complication rate was $21 \%$. However, it should be kept in mind that this study only employed two kinds of oncoplastic techniques. As technical varieties and experience increase, more patients will be eligible for OPS, and oncologic safety will improve.

DeLorenzi et al. [21] compared OPS and BCS techniques for pure DCIS and reported surgical margin positivity rates as 4.5 and $3.5 \%$, respectively. The IBTR rates have been found as similar between the groups. The IBTR rates increase in parallel with the increased follow-up time. This study shows that OPS can improve breast conservation rates.

Clough et al. [22] suggested that revision surgery is required for $30-60 \%$ of patients who have undergone BCS for DCIS because of bad cosmetic outcomes. It has been suggested that level II oncoplastic techniques can increase specimen volume besides increasing the surgical margin width by $20 \%$.

In reduction mammoplasty surgeries, a wider glandular tissue mobilization and replacement is performed when compared to other level II techniques. In this case, a common concern is that the surgical clips in the tumor bed may displace, which will decrease the efficacy of the radiotherapy boost dose. Eaton et al. [23] evaluated reduction mammoplasties for invasive and in situ tumors and reported that even though the surgical clips were outside the primary tumor quadrant in $43 \%$ of the patients, the 5 -year local control rates for IBTR were $91 \%$ for DCIS and 93\% for invasive tumors. The authors suggest that irradiating the primary tumor quadrant with boost dose will provide better local control, irrespective of the clips in patients who have undergone reduction mammoplasty.

In the literature, only a small number of studies which have investigated OPS techniques in surgical treatment of DCIS have evaluated early morbidity. In our study, even though the wound complication rates were higher than patients who have undergone BCS, all of these were minor complications. These minor wound complications after OPS did not affect the length of hospital stay and the starting time of adjuvant treatment. The limitations of our study were the retrospective design, relatively short follow-up period, and lack of evaluation of cosmetic results.

\section{Conclusion}

While there are numerous studies in the literature on OPS for invasive tumors, the number of studies investigating the oncological outcomes and safety of OPS for DCIS is extremely low. Studies regarding OPS for DCIS 
are mostly retrospective single-center studies that involve a small number of patients $[18,20]$. Even invasive tumors involving in situ components are also included in the analysis in some studies. To the best of our knowledge, the French study [10] with 68 patients is the largest series on this subject. The European Institute of Oncology study [21] is the only series that assigned BCS-performed patients as the control group. This study involves patients from 2000 to 2008, to whom level 1 or 2 techniques were performed as OPS. Our series is valuable because it evaluates only patients with pure DCIS, has a control group for comparison, and only consists of patients in whom level 2 techniques are performed. Considering that only $31 \%$ of our patients undergoing mastectomy had extensive MCs in our series, this study provided evidence that OPS can be used safely in the surgical treatment of DCIS by reducing re-excision and completion mastectomy rates compared to BCS.

\section{Statement of Ethics}

Ethics committee approval was received with number $\mathrm{AOH}-$ 42-2012. As it was a retrospective study, it was not necessary to obtain informed consent from the patients.

\section{Disclosure Statement}

The authors declare no conflicts of interest.

\section{Funding Sources}

None.

\section{Author Contributions}

Planning of study, data collection, evaluation, and writing of the article: Lutfi Dogan, Mehmet Ali Gulcelik.

\section{References}

1 Weigel S, Khil L, Hense HW, Decker T, Wellmann J, Heidrich J, et al. Detection Rates of Ductal Carcinoma in Situ with Biennial Digital Mammography Screening: Radiologic Findings Support Pathologic Model of Tumor Progression. Radiology. 2018 Feb;286(2): 424-32.

2 Tang P, Hajdu SI, Lyman GH. Ductal carcinoma in situ: a review of recent advances. Curr Opin Obstet Gynecol. 2007 Feb;19(1): 63-7.

3 Love SM, Barsky SH. Breast-duct endoscopy to study stages of cancerous breast disease. Lancet. 1996 Oct;348(9033):997-9.

4 Silverstein MJ, Lagios MD, Groshen S, Waisman JR, Lewinsky BS, Martino S, et al. The influence of margin width on local control of ductal carcinoma in situ of the breast. N Engl J Med. 1999 May;340(19):1455-61.

5 Jeevan R, Cromwell DA, Trivella M, Lawrence G, Kearins O, Pereira J, et al. Reoperation rates after breast conserving surgery for breast cancer among women in England: retrospective study of hospital episode statistics. BMJ. 2012 Jul;345 jul12 2:e4505.

6 Weber WP, Soysal SD, El-Tamer M, Sacchini V, Knauer M, Tausch C, et al. First international consensus conference on standardization of oncoplastic breast conserving surgery. Breast Cancer Res Treat. 2017 Aug;165(1): 139-49.

7 McCulley SJ, Durani P, Macmillan RD. Therapeutic mammaplasty for centrally located breast tumors. Plast Reconstr Surg. 2006 Feb; 117(2):366-73.

8 Ernster VL, Ballard-Barbash R, Barlow WE, Zheng Y, Weaver DL, Cutter G, et al. Detection of ductal carcinoma in situ in women undergoing screening mammography. J Natl Cancer Inst. 2002 Oct;94(20):1546-54.
9 Faverly DR, Burgers L, Bult P, Holland R. Three dimensional imaging of mammary ductal carcinoma in situ: clinical implications. Semin Diagn Pathol. 1994 Aug;11(3):193-8.

10 van la Parra RF, Clough KB, Lejalle-Alaeddine C, Poulet B, Sarfati I, Nos C. Oncoplastic Level 2 Mammoplasty for Large DCIS: 5-Year Results. Ann Surg Oncol. 2019 Aug;26(8): 2459-65.

11 Correa C, McGale P, Taylor C, Wang Y, Clarke M, Davies C, et al.; Early Breast Cancer Trialists' Collaborative Group (EBCTCG). Overview of the randomized trials of radiotherapy in ductal carcinoma in situ of the breast. J Natl Cancer Inst Monogr. 2010; 2010(41):162-77.

12 Cochrane RA, Valasiadou P, Wilson AR, AlGhazal SK, Macmillan RD. Cosmesis and satisfaction after breast-conserving surgery correlates with the percentage of breast volume excised. Br J Surg. 2003 Dec;90(12):1505-9.

13 Losken A, Elwood ET, Styblo TM, Bostwick J 3rd. The role of reduction mammaplasty in reconstructing partial mastectomy defects. Plast Reconstr Surg. 2002 Mar;109(3):968-75.

14 Morrow M, Van Zee KJ, Solin LJ, Houssami N, Chavez-MacGregor M, Harris JR, et al. Society of Surgical Oncology-American Society for Radiation Oncology-American Society of Clinical Oncology Consensus Guideline on Margins for Breast-Conserving Surgery with Whole-Breast Irradiation in Ductal Carcinoma In Situ. Ann Surg Oncol. 2016 Nov;23(12):3801-10.

15 Coates AS, Winer EP, Goldhirsch A, Gelber RD, Gnant M, Piccart-Gebhart M, et al.; Panel Members. Tailoring therapies - improving the management of early breast cancer: St Gallen International Expert Consensus on the Primary Therapy of Early Breast Cancer 2015. Ann Oncol. 2015 Aug;26(8):1533-46.
16 Melstrom LG, Melstrom KA, Wang EC, Pilewskie M, Winchester DJ. Ductal carcinoma in situ: size and resection volume predict margin status. Am J Clin Oncol. 2010 Oct; 33(5):438-42.

17 King TA, Sakr R, Patil S, Gurevich I, Stempel M, Sampson M, et al. Clinical management factors contribute to the decision for contralateral prophylactic mastectomy. J Clin Oncol. 2011 Jun;29(16):2158-64.

18 Szynglarewicz B, Maciejczyk A, Forgacz J, Matkowski R. Breast segmentectomy with rotation mammoplasty as an oncoplastic approach to extensive ductal carcinoma in situ. World J Surg Oncol. 2016 Mar;14(1):72.

19 Tadros AB, Smith BD, Shen Y, Lin H, Krishnamurthy S, Lucci A, et al. Ductal carcinoma in situ and margins $<2 \mathrm{~mm}$ : contemporary outcomes with breast conservation. Ann Surg. 2019 Jan;269(1):150-7.

20 Song HM, Styblo TM, Carlson GW, Losken A. The use of oncoplastic reduction techniques to reconstruct partial mastectomy defects in women with ductal carcinoma in situ. Breast J. 2010 Mar-Apr;16(2):141-6.

21 De Lorenzi F, Di Bella J, Maisonneuve P, Rotmensz N, Corso G, Orecchia R, et al. Oncoplastic breast surgery for the management of ductal carcinoma in situ (DCIS): is it oncologically safe? A retrospective cohort analysis. Eur J Surg Oncol. 2018 Jul;44(7):957-62.

22 Clough KB, van la Parra RF, Thygesen $\mathrm{HH}$, Levy E, Russ E, Halabi NM, et al. Long-term results after oncoplastic surgery for breast cancer: a 10-year follow-up. Ann Surg. 2018 Jul;268(1):165-71.

23 Eaton BR, Losken A, Okwan-Duodu D, Schuster DM, Switchenko JM, Mister D, et al. Local recurrence patterns in breast cancer patients treated with oncoplastic reduction mammaplasty and radiotherapy. Ann Surg Oncol. 2014 Jan;21(1):93-9. 